\title{
Analysis of Focus Errors in Lithography using Phase-Shift Monitors
}

\author{
Bruno La Fontaine ${ }^{* a}$, Mircea Dusa ${ }^{* * b}$, Jouke Krist $^{\mathrm{b}}$, Alden Acheta ${ }^{\mathrm{a}}$, Jongwook Kye ${ }^{\mathrm{a}}$, \\ Harry Levinson ${ }^{\mathrm{a}}$, Carlo Luijten ${ }^{\mathrm{b}}$, Craig Sager ${ }^{\mathrm{c}}$, Jack Thomas ${ }^{\mathrm{a}}$, Judith Van Praagh ${ }^{\mathrm{b}}$ \\ ${ }^{\mathrm{a}}$ Advanced Micro Devices; ${ }^{\mathrm{b}} \mathrm{ASM}$ Lithography; ${ }^{\mathrm{c}}$ Benchmark Technology
}

\begin{abstract}
We present here the characterization of focus errors on a 193-nm scanner using phase-shift focus monitors, which clearly identifies the influence of full field dynamic effects and that of the wafer topography. These results are used to correct the systematic errors due to tool set-up. We also present results using a new focus monitor based on phase gratings, which is more sensitive than the traditional phase-shift focus monitor.
\end{abstract}

Keywords: Lithography, Focus, Phase-Shift Focus Monitor,

\section{INTRODUCTION}

As the dimensions of devices shrink, the numerical aperture of the lithography scanners increases and the actinic wavelength decreases. This leads to a reduction of the useable depth of focus for lithography printing, as is illustrated in Figure 1. In this graph, the imaging depth of focus is derived from the Rayleigh criterion and is plotted against time, assuming that the CD will follow the ITRS Roadmap for DRAM half-pitch and that ArF, $\mathrm{F}_{2}$ and EUV Lithography are introduced respectively in 2004, 2007 and 2010.

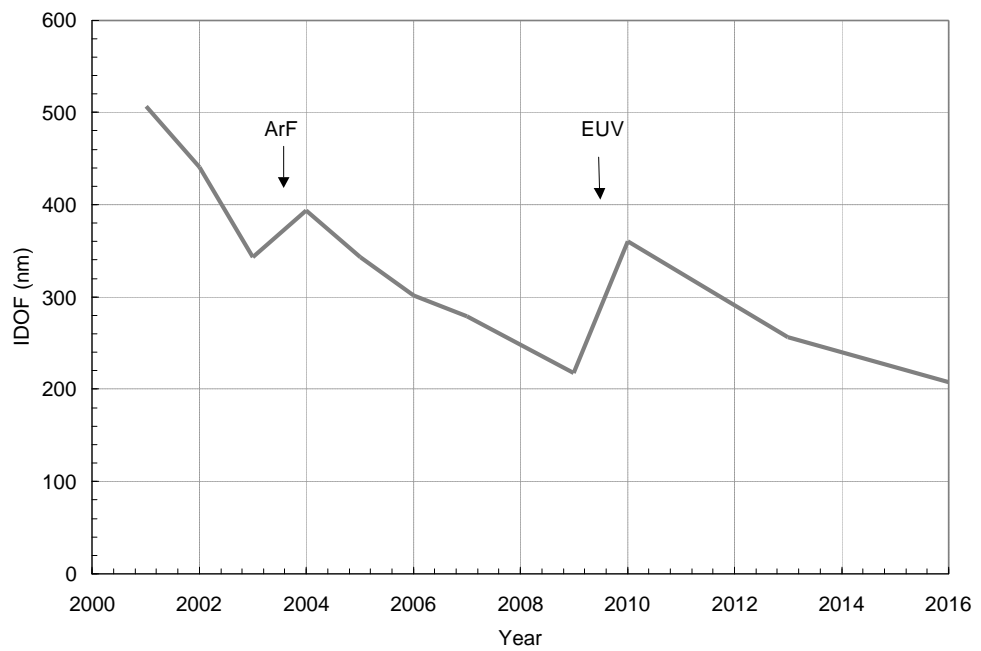

Figure 1 - Illustration of how the imaging depth of focus (IDOF) might be shrinking over time, as the actinic wavelength gets shorter and the numerical aperture NA gets larger. We assume that the resolution will follow the DRAM half-pitch values published in the 2001 ITRS Roadmap. We also assumed that ArF lithography would be inserted around 2003-2004, that F2 lithography would happen around 2007 and EUVL around 2010 .

In order to maintain process capability, one needs to be able to characterize the distribution of focus errors produced by the scanner with high sensitivity and good repeatability. In particular, one would like to identify systematic errors associated with the tool or the process used in order to correct them and minimize their contribution to the focus budget.

\footnotetext{
*bruno.lafontaine@amd.com; phone 1 (408) 749-2060; fax 1 (408) 774-8813; http://www.amd.com; Advanced Micro Devices, One AMD Place, m/s 78, PO Box 3453, Sunnyvale, CA 94088-3453;

mircea.dusa@asml.com; phone 1 (408) 855-0516; fax 1 (408) 855-0549; http://www.asml.com; ASM Lithography, 4800 Great America Parkway, Suite 400,Santa Clara, CA 95054 USA;
} 
Many different methods can be used to evaluate focus errors at the image plane of a lithography tool. We describe here just a few of the most popular methods that use resist as a detector. For instance, using a focus matrix on a wafer, one can identify the best focus setting by identifying at which focus offset the smallest features appear in resist ${ }^{2}$, or by measuring the latent image contrast as a function of focus offset ${ }^{3}$. Other more empirical techniques based on the visual observation of dot arrays through focus can be used. ${ }^{4}$ Using a focus-exposure matrix (FEM), one can generate a family of curves representing linewidth change through focus, which is called a Bossung plot. ${ }^{5}$ From these plots, the best focus and depth of focus can be estimated for different type of features. Finally, the use of phase-shift masks, introduced in 1993 by Brunner ${ }^{6,7}$, provides a means for inferring focus errors from changes in the position of the image in the resist.

In this paper, we describe in section 2 two of the techniques that were used to monitor the focus error distribution on our tools, the phase-shift focus monitor (PSFM) and the phase-shift grating focus monitor (PGFM), which is a new monitor that is more sensitive than the usual PSFM. In section 3, we use these focus monitors to measure and identify the various sources of focus errors contributing to this distribution and show how the analyzed data can be used to minimize some of the contributions.

\section{MEASUREMENT TECHNIQUES}

\subsection{Phase-Shift Focus Monitor (PSFM)}

The focus monitor patterns that we used for the present study ${ }^{\text {Error! Reference source not found. }}$ are shown schematically in Figure 2. The basic operating principle of this test is that the aerial image of a line with a $90^{\circ}$-phase patch on one of its side tends to move toward this patch when there is a positive defocus and away from it for negative defocus. When the lines and phase patches are arranged so as to form concentric boxes, any focus error is translated into an alignment error that can be measured with an overlay measurement tool.

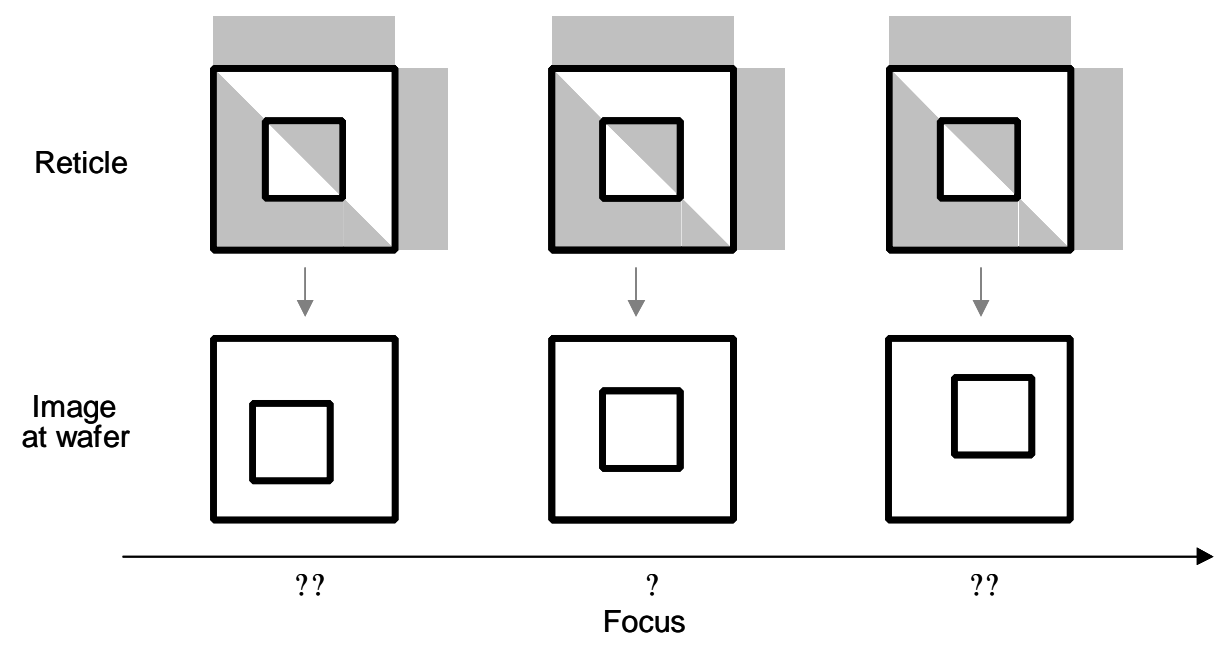

Figure 2 - The focus monitor, as used in this study. Two concentric boxes, each made of a small line, have $90^{\circ}$ phase patches adjacent to portions of their perimeter. As the focus drifts away from its best setting, the lines that make up the frames shift toward or away from their nominal position, depending on the sign of the focus error. This results in an alignment error that can be captured with an overlay measurement tool.

A plot of the measured overlay as a function of programmed defocus is then used to quantitatively evaluate any focus errors. Sample calibration curves, for features oriented horizontally and vertically, are plotted in Figure 3 below. These curves were obtained by imaging boxes composed of 160-nm lines on a 193-nm scanner, with a pupil fill factor of $?=0.321$. The two curves cross at a programmed defocus value of about $+200 \mathrm{~nm}$, indicating that the best-focus image of these lines is obtained at a focus setting of $+200 \mathrm{~nm}$. 


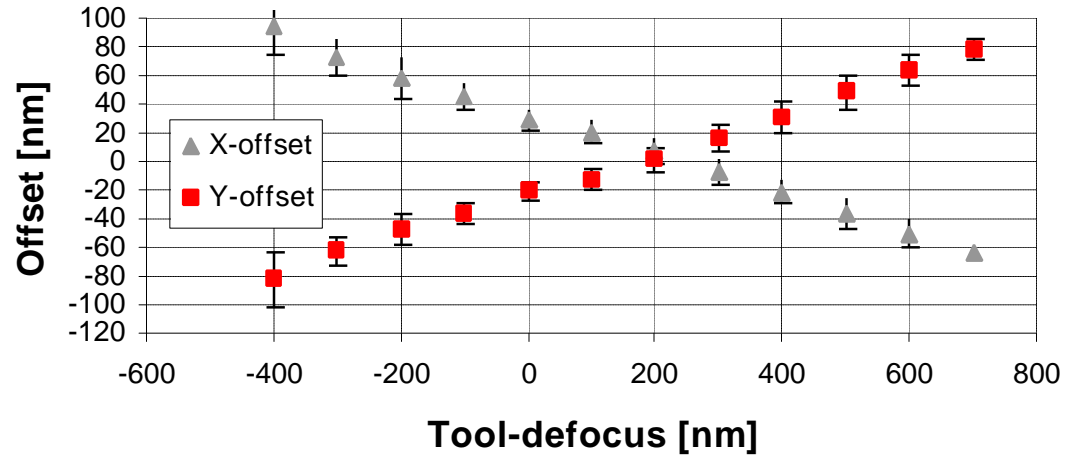

Figure 3 - Sample calibration curves obtained with a 193-nm tool, with ? ?=0.321. The lines making up the box patterns had a width of $160 \mathrm{~nm}$.

The slope of the calibration curve in Fig. 3, is $7.14 \mathrm{~nm}$ defocus/nm overlay. Since there are 7 columns of PSFM marks across the slit and 11 marks along the scan direction for each column, a total of 77 measurements can be made across the scanned image field, resulting in 77 slope measurements. The set of slope measurement has a distribution that is presented in Figure 4. This slope distribution is fairly uniform across the scanned field and has a standard deviation of 4 to $5 \%$.

X-slope (nm defocus/nm overlay) across the field

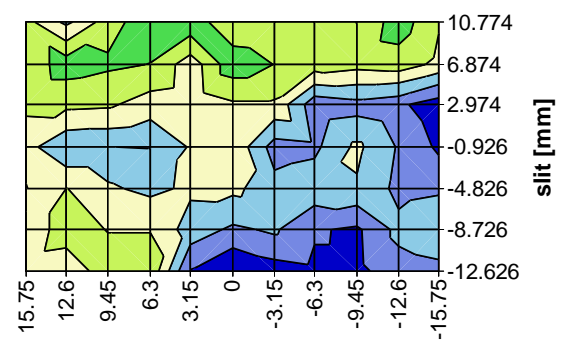

scan $[\mathrm{mm}]$
Y-slope (nm defocus/nm overlay) across the field

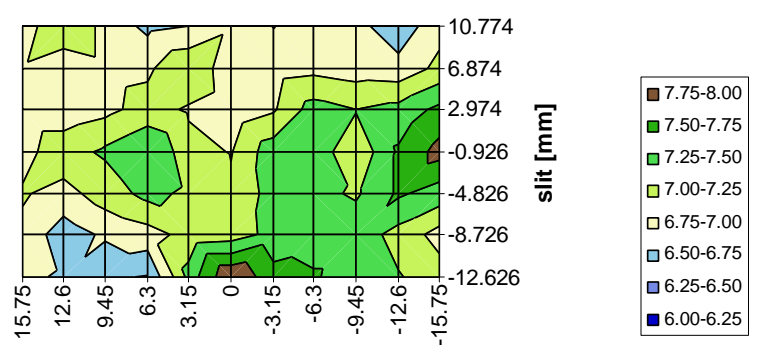

scan $[\mathrm{mm}]$

Figure 4 - Slope variation across the field for X-and Y-orientation. The slope variation across the field, for the X-and Y-orientation shows a good uniformity, with a distribution characterized by a standard deviation of ? $4 \%$.

In the analyses presented in Section 3, we used an average slope value to determine how focus errors were distributed across the scanned image field and across the entire wafer.

\subsection{Phase-grating focus monitor (PGFM)}

The phase-grating focus monitor is based on the same idea of converting focus errors into alignment errors and measuring them with an overlay measurement tool. However, the isolated lines with the large $90^{\circ}$ phase patches used for the PSFM are replaced with phase gratings, introduced by Nomura ${ }^{8}$, which generate the equivalent of a two-beam interferometer inside the lens and are very sensitive to focus errors. The patterns used for the focus measurements are depicted in Figure 5. 


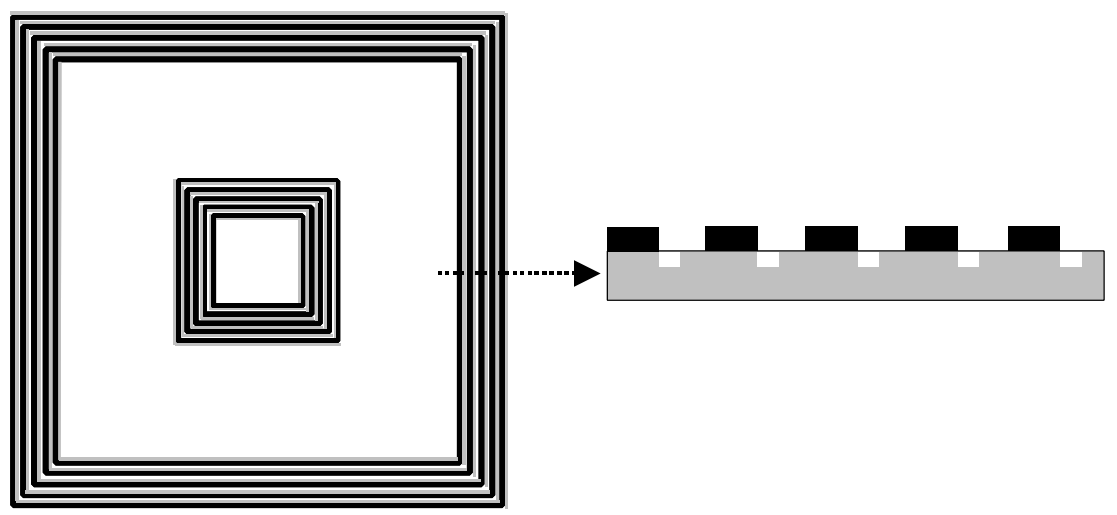

Figure 5 - The phase-grating focus monitor (PGFM). The basic pattern is made of two concentric boxes composed of phase gratings. On the left-side picture, black lines represent $\mathrm{Cr}$ and gray lines are $90^{\circ}$ phase lines etched in the glass. A lineout through a portion of the pattern is shown on the right side (with Cr black and glass gray) in order to show the phase grating in more detail.

A calibration curve of the PGFM is plotted in Figure 6. This curve was obtained by imaging boxes composed of 500-nm pitch gratings on the same 193-nm scanner as that used for Fig. 3, also with a pupil fill factor of $?=0.321$. The average slope of the calibration curves across the field for these conditions is $3.03 \mathrm{~nm}$ defocus $/ \mathrm{nm}$ overlay, which is more than twice as sensitive to focus errors that the PSFM. The slope distribution was also fairly uniform across the field, with a standard deviation of ? 8\%. Most of the observed slope variation was due to field non-uniformities. When this test was done again on a better machine, the distribution of slopes had a standard deviation of less than $5 \%$.

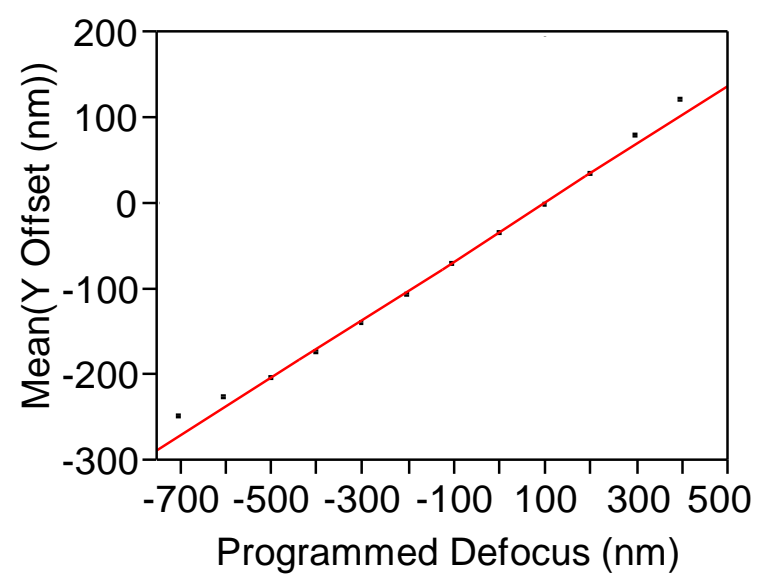

Figure 6 - Sample calibration curves obtained with a 193-nm tool, with ? ? 0.321 . The gratings making up the box patterns had a pitch of $500 \mathrm{~nm}$.

\subsection{Experimental conditions}

For the experiments reported in this paper, we used an ArF step-and-scan tool, with a numerical aperture of NA $=0.63$. The illumination was set at $?=0.321$. We used PAR707 resist on an AR19 anti-reflective coating, and the dose used was $11.4 \mathrm{~mJ} / \mathrm{cm}^{2}$.

\section{RESULTS AND ANALYSIS}

In this section we will present the characterization results from this tools, look at how the results could be used to correct scanning errors and level sensing problems. We also present an analysis of the influence of the wafer topography on the focus errors. Finally, we examine differences in the results obtained with PSFM and PGFM patterns. 


\subsection{Whole wafer focus distribution}

Using the PSFM reticle, we exposed full wafers at constant focus with 31 fields measuring $27 \mathrm{~mm}$ ? $33 \mathrm{~mm}$, in order to evaluate the total distribution of focus errors. A contour map of the focal plane deviation, as defined by FPD $=\left(z_{x}+z_{y}\right) / 2$ is plotted in Figure 7, where $z_{x(y)}$ is the focus offset determined from the $x(y)$ component of the PSFM pattern offset. Note the large focus deviations at the edge of the wafer.

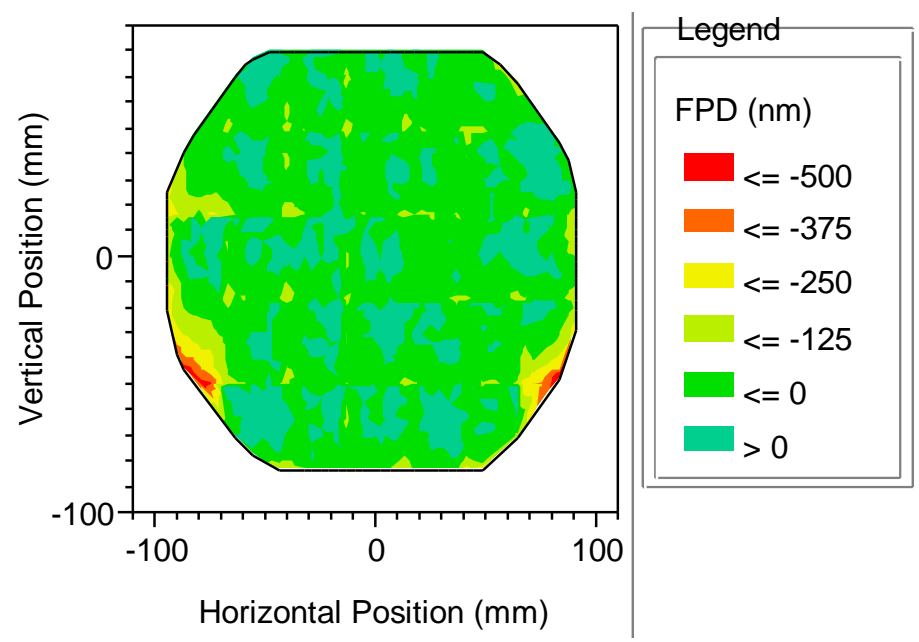

Figure 7 - Contour map of focus errors on a $200 \mathrm{~mm}$ wafer exposed at constant focus. The outline of each individual field can be perceived, indicating that the average field errors represent a sizeable contribution to the total distribution of focus errors. Note also the large focus deviations at the edge of the wafer.

The distribution of focus errors measured on this wafer is presented in Figure 8. The standard deviation estimated of this distribution is $?=84 \mathrm{~nm}$. Since the distribution of focus displays a non-Gaussian shape, it is likely that systematic errors contribute a fair fraction of the total range.

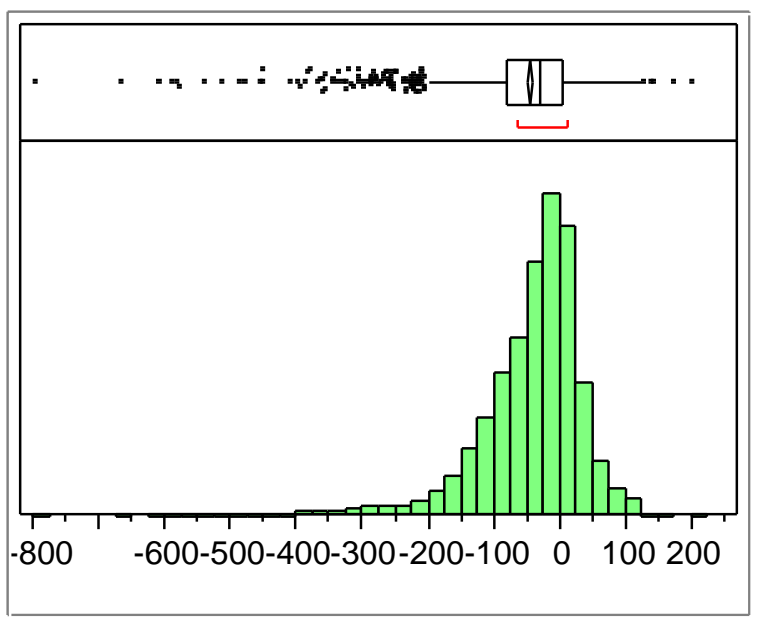

Figure 8 - Distribution of all focus errors measured using a constantfocus exposure. The data is the same as that presented in Figure 7. Note the non-Gaussian shape of the distribution, which indicates that systematic errors are sizeable. The standard deviation calculated from this data is $?=84 \mathrm{~nm}$.

In order to evaluate the importance of systematic error, we examine next the focus distribution across the average field. 


\subsection{Systematic errors - average field}

For each position within the image field, we averaged the data from all fields of a constant-focus exposure wafer. The results are presented in Figure 9. Note the significant contribution from scanning errors.
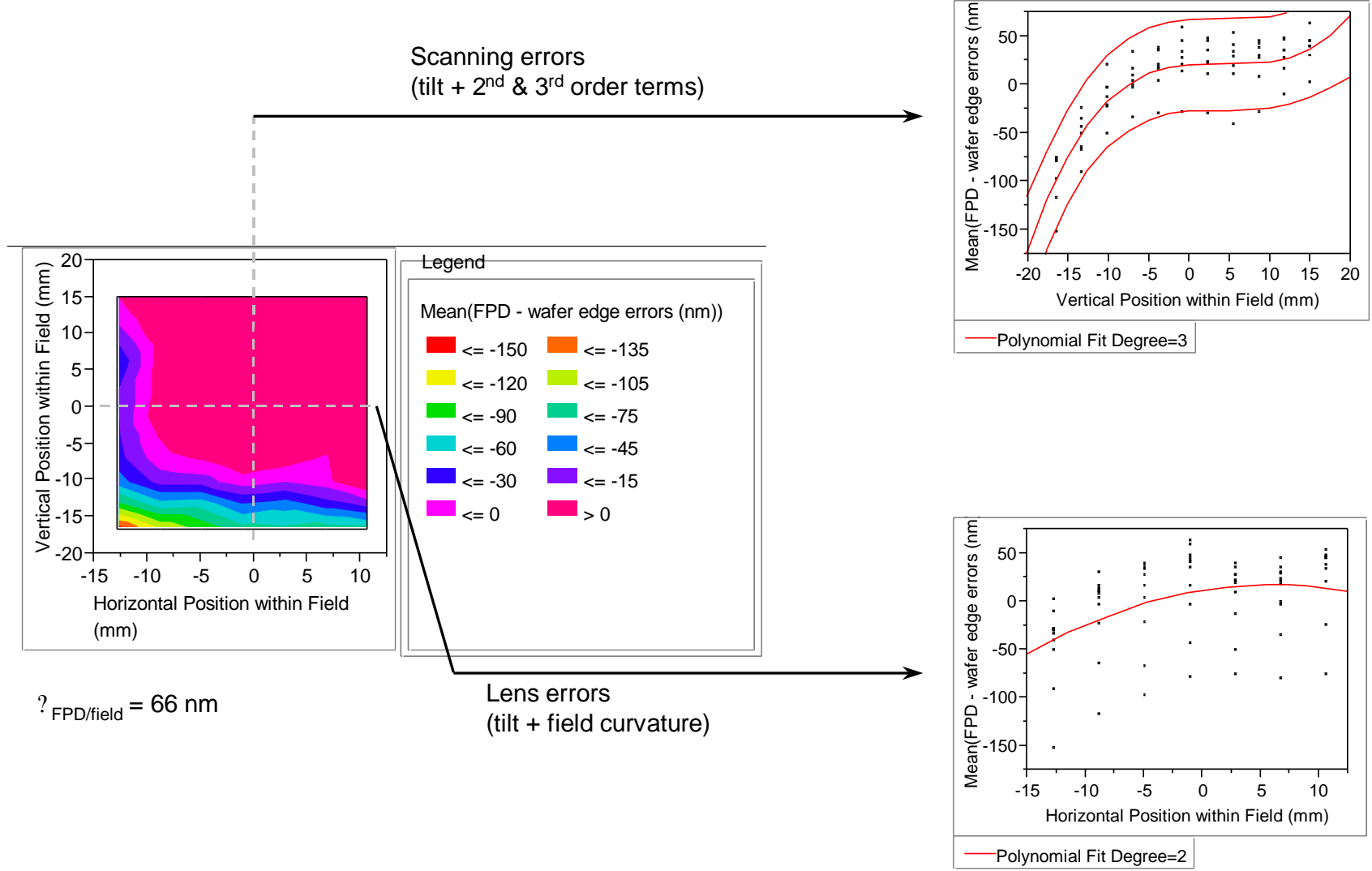

Figure 9 - Distribution of focal plane deviation for the average field. (a) Contour map of FPD. (b) FPD along the slit, including all positions along the scan. (c) FPD along the scan direction, including all positions along the slit.

Based on these data, we adjusted the tilt of the reticle stage in order to minimize the large FPD non-uniformity along the scan direction. The results in Figure 10 illustrate well the improvement that was achieved. The impact on the total FPD distribution across the wafer is also reported below in Figure 11(c). 


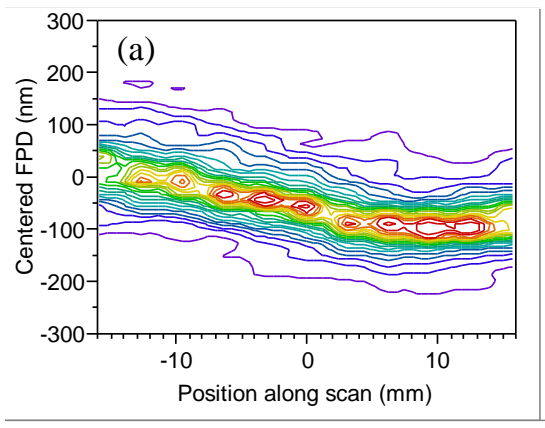

.1 .2 .3 .4 .5 .6 .7 .8 .9 Quantile Density Contours

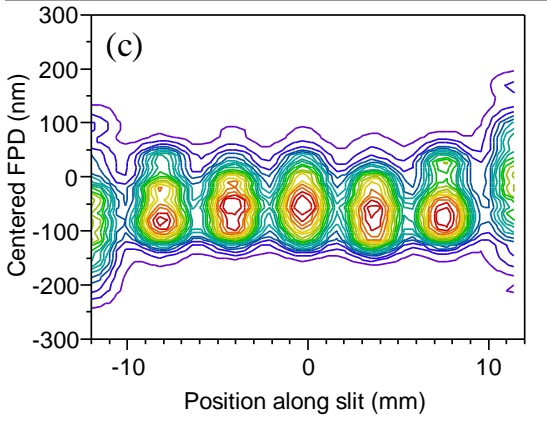

$\overline{1.2 .3 .4 .5 .6 .7 .8 .9}$ Quantile Density Contours

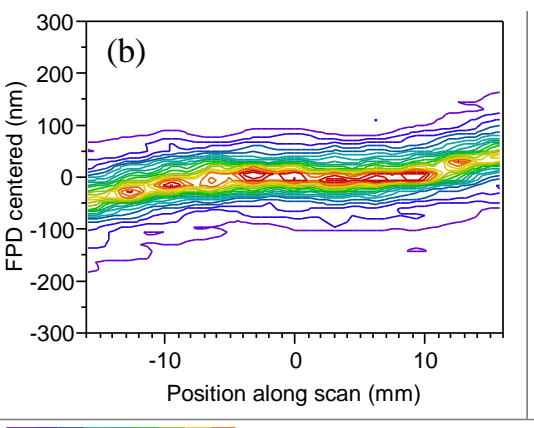

$\overline{1.2 .3 .4 .5 .6 .7 .8 .9}$ Quantile Density Contours

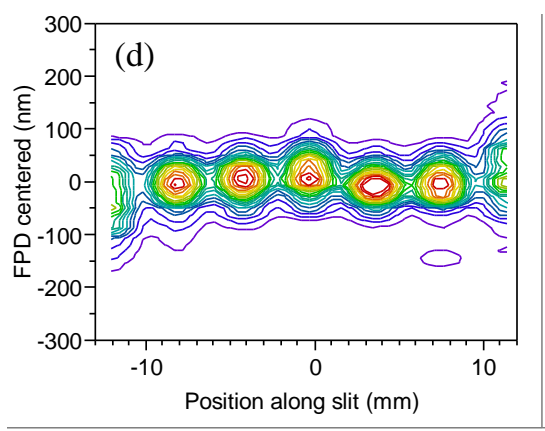

$\overline{1.2 .3 .4 .5 .6 .7 .8 .9}$ Quantile Density Contours
Figure 10 - Reticle stage adjustments showing improved FPD characteristics. (a)(c) FPD along the scan and slit direction before the reticle stage adjustment (b)(d) FPD along the scan and slit after adjustment of the reticle stage. One can clearly observe the tighter distribution of FPD after the reticle stage tilt was optimized.

\subsection{Systematic errors - field to field}

From the FPD contour map shown in Figure 7, we could tell that there were large errors at the edge of the field. These errors can be traced back to unexpected scan directions (i.e. from the outside to the inside of the wafer) used on some of the edge dice (6) for these exposures. This means that the level sensors for setting the focus $z$ and the tilt could not be used for these dice and settings from adjacent dice were used instead. By modifying the job we got rid of most of the dice where the level sensors could not be used; only two remained instead of six. The contour maps of FPD for the original case and the optimised case are compared in Figure 11(a) and 11(b). These results show a significant improvement in the total FPD when the optimised settings, as determined by the focus monitor, are used. When the optimised settings for levelling and tilt of the reticle stage are used together, the total focus error is reduced by a factor of about two compared to the original settings (Figure 11(c)). 

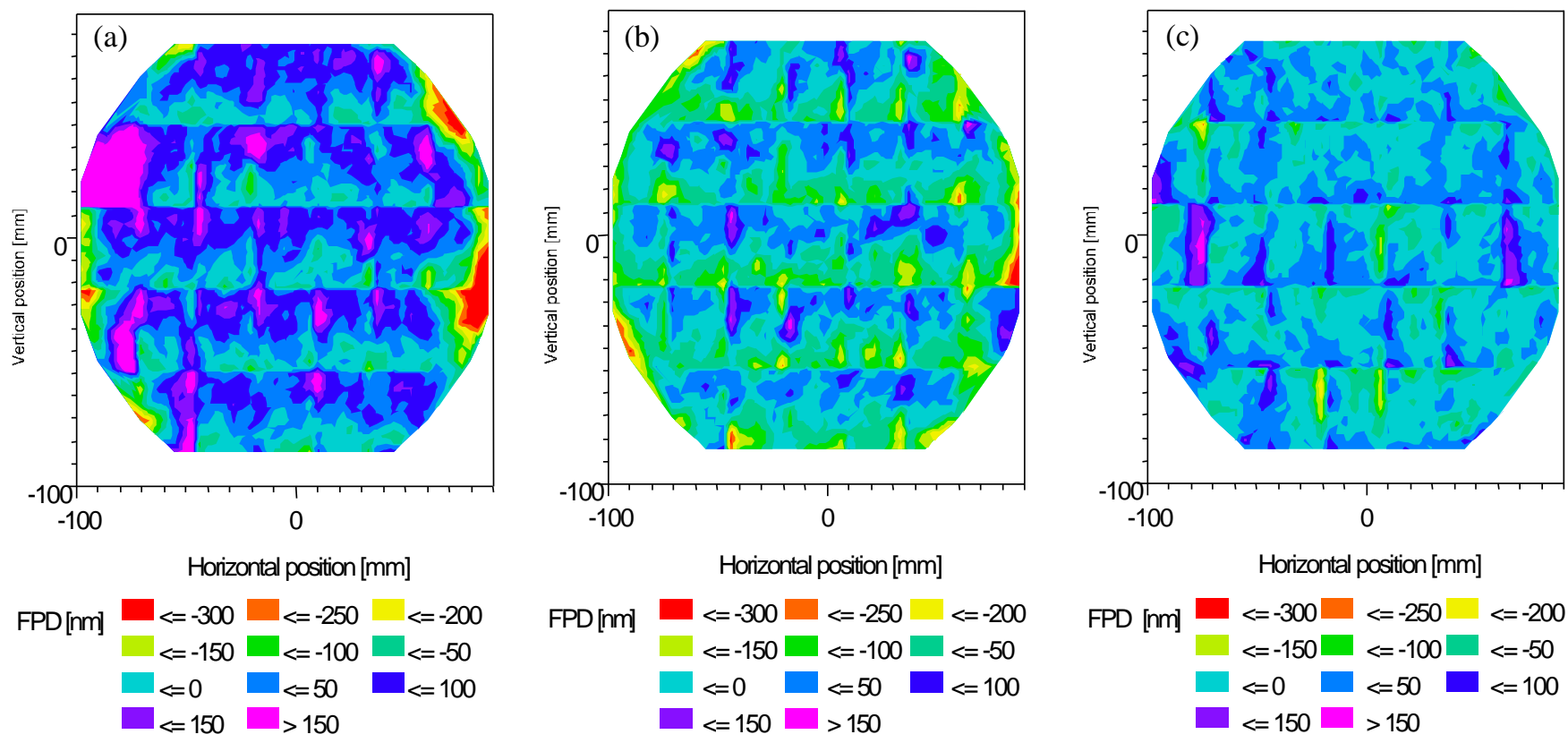

Figure 10 - Contour maps of wafer focus distribution. (a) Original state: the reticle stage tilt was not optimized and the level sensors were not used for 6 fields at the edge of the wafer, resulting in a FPD standard deviation of ? = 100 nm. (b) Optimized leveling. The resulting standard deviation is $?=\mathbf{7 6} \mathrm{nm}$. (c) Optimized leveling and adjustment to the reticle stage tilt. The resulting standard deviation is $?=\mathbf{5 4} \mathrm{nm}$.

\subsection{Wafer flatness contribution and residual errors}

Next, we tried to quantify the contribution of wafer flatness to the total FPD distribution across a printed wafer. For this purpose, we used wafers of different flatness quality and measured how the range of FPD varied across these wafers. The wafer flatness was characterized by a capacitive gauge sensor with a 4-mm head and the range of deviation from the best plane over multiple areas of $25 \mathrm{~mm}$ ? $25 \mathrm{~mm}$ was reported for each of these areas. The sum of the mean value and of three times the standard deviation $($ mean +3 ? ) for all fields on a wafer was then used to describe the flatness of that wafer.

The results are presented in Figure 11. The red circles correspond to 6? value of the raw distribution of FPD for each wafer and the blue circles represent the 6? value of the distribution of FPD after correcting for systematic errors such as tilt along the slit and along the scan. One can see that the wafer flatness contributes significantly to the total distribution of focus errors unless the wafer flatness is smaller than about $200 \mathrm{~nm}$. Also, we can conclude from this analysis that the residual errors contribute between 200 and $250 \mathrm{~nm}$ to the total FPD distribution. 


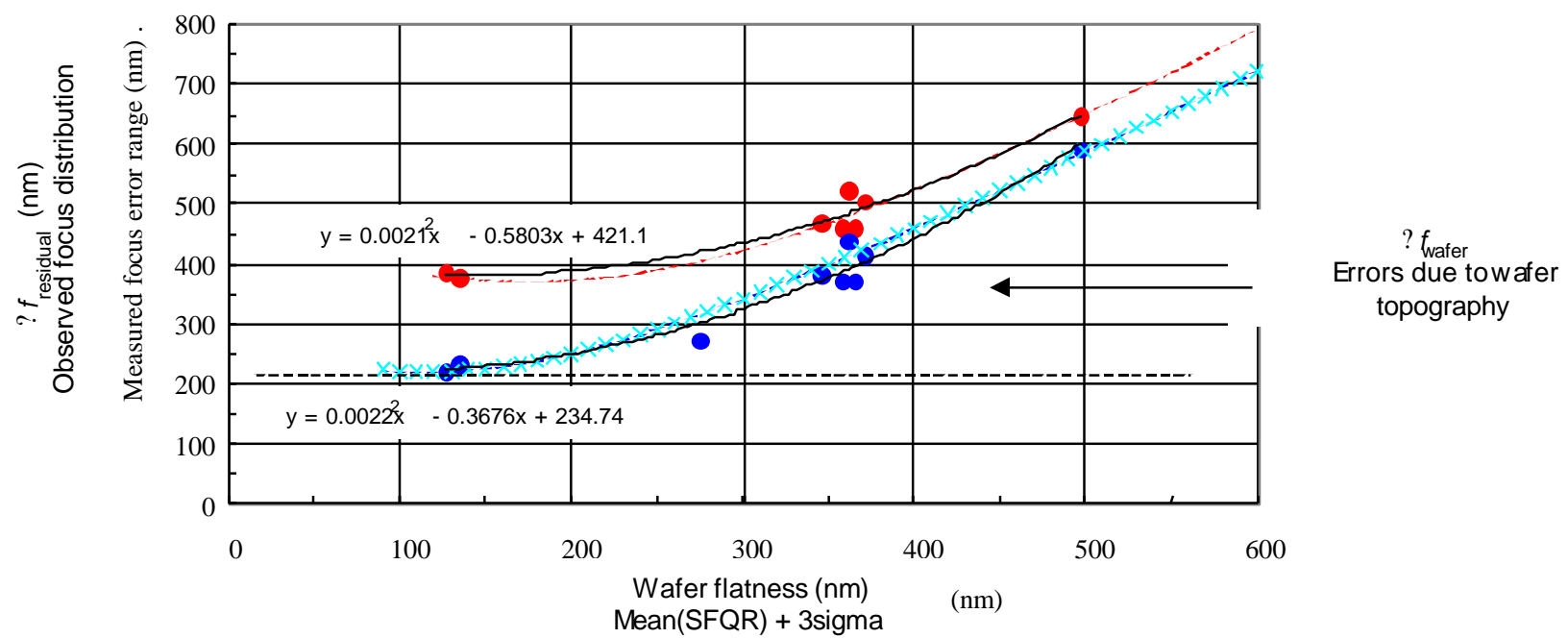

Figure 11 - Influence of wafer flatness on the focus error distribution. The red circles correspond to 6 ? value of the raw distribution of FPD for each wafer and the blue circles represent the 6? value of the distribution of FPD after correcting for systematic errors. For wafers with flatness better than about $200 \mathrm{~nm}$, the focus errors are dominated by the tool and by residual errors.

\subsection{Differences between PSFM and PGFM results}

Finally, it is worthwhile pointing out some of the differences that could be observed in the analysis of the PSFM and PGFM data. Comparing Figs.3 and 6, one may note the intercept of the calibration curves with the zero offset line is different. The PSFM indicates that the best focus is at $z=200 \mathrm{~nm}$, while PGFM predicts best focus at $z=100 \mathrm{~nm}$. This can be explained in the way the image-spectrum of these features interacts with the tool aberrations.

This is illustrated in Figure 12, where the spectrum of the PSFM and PGFM features, as projected in the pupil plane, are plotted along with a phase error curve corresponding to spherical aberrations. On the left, the energy projected by the PSFM feature is spread out more evenly through the pupil, while the PGFM (on the right) produces a sharp peak at about two thirds of the NA. We know that the tool being tested here has a significant amount of spherical aberrations. The predicted focus offset between these two features due to spherical aberrations is indeed $100 \mathrm{~nm}$, as measured here. ${ }^{10}$ Since the aberrations are not constant across the field on this tool, a difference in the FPD tilt value is also observed experimentally. 

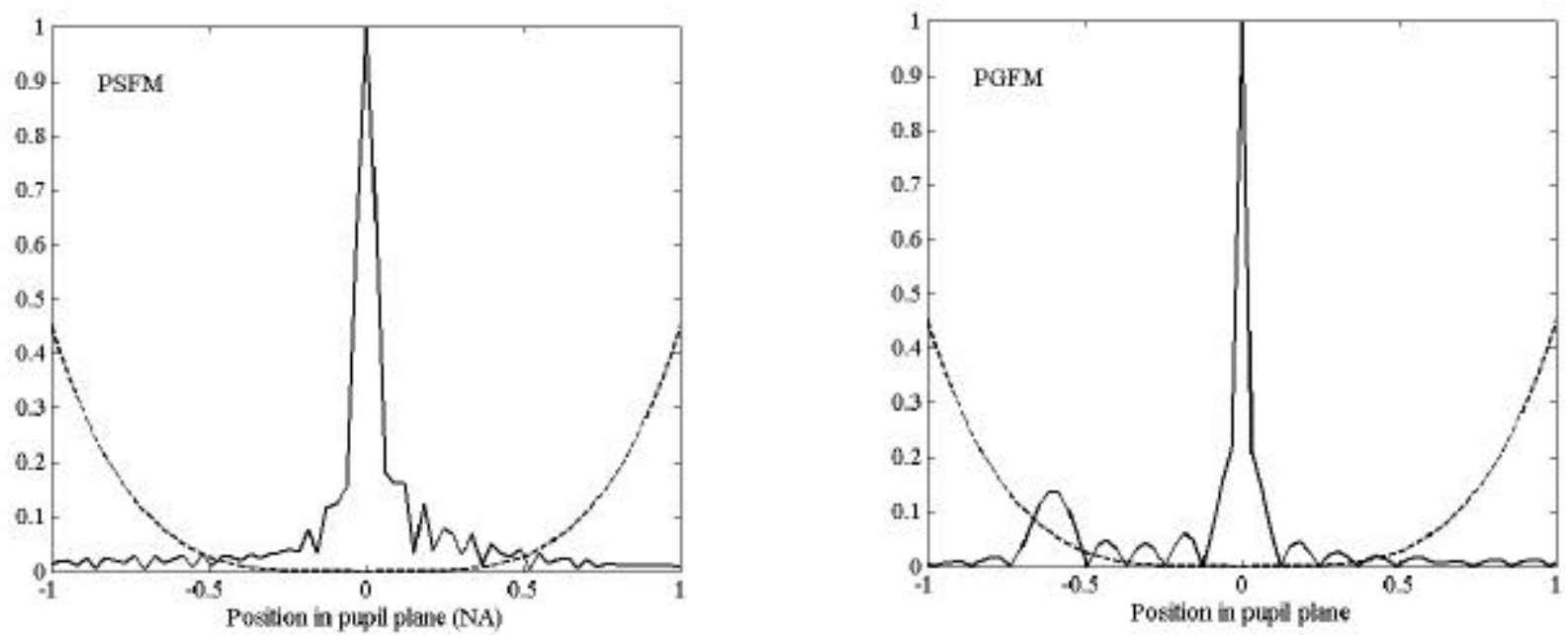

Figure 11 - Interaction of tool aberration with the image forming mechanism for both monitor. The solid lines represent the spectrum of the structures used for PSFM (left) and PGFM (right). The dashed lines represent some level of spherical aberrations. Since the structures used by the two monitors probe the pupil of the tool differently, they have a different sensitivity to different aberrations. Because of known spherical aberrations in the tool under test, it is predicted that there should be a $100 \mathrm{~nm}$ focus offset between the two focus monitors.

\section{CONCLUSION}

We have shown clearly that the phase-shift focus monitors can be used to optimize a lithography tool, which can be used to keep a process under control. We were also able to measure the influence of the wafer flatness on the focus errors and infer the magnitude of the residual focus errors on a 193-nm step-and-scan tool.

We presented a new monitor, PGFM, based on phase-grating patterns that can be more sensitive to focus errors than the standard PSFM. This new monitor was implemented successfully to evaluate the performance of the ArF tool. This type of improvement in the sensitivity of focus monitors will be particularly useful as tools have smaller and smaller depths of focus and the focus control needs to improve.

\section{ACKNOWLEDGMENTS}

The authors would like to acknowledge many useful discussions with various members of AMD's Process Development Lithography Department. 


\section{REFERENCES}

1. International Technology Roadmap for Semiconductors (ITRS), http://public.itrs.net.

2. J. Kirk, "Astigmatism and field curvature from pin-bars", Proc. SPIE, Vol. 1463 (1991), p.282.

3. T. Adams, "Applications of latent image metrology in microlithography", Proc. SPIE, Vol. 1463 (1991), p.294.

4. S. Stalnaker et al., "Focal plane determination for sub-half micron optical steppers", Microelectronic Engineering 21 (1993), p.33.

5. Reference for Bossung plots...

6. T.A. Brunner, "New focus metrology technique using special test mask", OCG Interface '93, Sept. 26, 1993, San Diego, CA. Reprinted in Microlithography World, 3 (1) (Winter 1994), pp. 5-13.

7. T.A. Brunner, A.L. Martin, R.M. Martino, C.P. Ausschnitt, T.H. Newman, "Quantitative stepper metrology using the focus monitor test mask", Proc. SPIE, Vol.2197 (1994), pp.541-549.

8. Benchmark Technologies, 7 Kimball Lane, Building E, Lynnfield MA 01940 USA, http://www.benchmarktech.com

9. H. Nomura, "New phase shift gratings for measuring aberrations", Proc. SPIE, Vol.4346 (2001), pp.25-35

10. J. Kye, private communication - The simulations were based on aberration values inferred from a Dirksen monitor and a modified Shack-Hartman test. 